\title{
ON THE WIND FLOW PATTERNS UNDER NEUTRAL AND UNSTABLE CONDITIONS IN AN URBAN AREA
}

\author{
Aristeidis A. Nezis ${ }^{1}$, Dionysios I. Angelidis ${ }^{2}$, Vasiliki D. Assimakopoulos ${ }^{3}$ \\ and George $C$. Bergeles ${ }^{2}$ \\ ${ }^{1}$ Department of Environmental Physics and Meteorology, Faculty of Physics, University of Athens, Greece \\ ${ }^{2}$ Department of Mechanical Engineering, National Technical University of Athens, Greece \\ ${ }^{3}$ National Observatory of Athens, Institute for Environmental Research and Sustainable Development, Greece \\ e-mail: vasiliki@meteo.noa.gr
}

\begin{abstract}
Wind flow, turbulence and pollutant dispersion characteristics within an urban street canyon depend largely on the thermal behaviour of the materials, e.g., the street floor under the sun and on the geometrical characteristics of the surrounding buildings under low wind conditions. They have a detrimental effect on the stability conditions within and above street canyons, which in turn affect the wind flow patterns and the local air quality. The purpose of the present work is to study experimentally and numerically the flow field developed under neutral and unstable stratification conditions within a typical square urban street canyon. A two-dimensional set up is employed including several canyons in order to simulate an urban environment. Experiments were carried out with the use of a triple hot sensor anemometer upwind above and downwind an isolated building. Measurements were taken under neutral stratification conditions for a wind speed of $3 \mathrm{~ms}^{-1}$. Two dimensional numerical simulations have also been conducted for typical urban street canyons. The numerical results have been obtained by the solution of the time averaged NavierStokes equations coupled with the standard $k-\varepsilon$ turbulence model. The performance of the CFD algorithm is increased by the unstructured Cartesian grid. The numerical code was firstly validated with the experimental results derived under neutral stratification conditions. The effects of stability within a typical urban area were also studied numerically. Spatial distributions of wind velocity, turbulence and pollutants, within street canyons, were analyzed under neutral and unstable conditions for Richardson number $R_{b}=-0.17$. Under unstable conditions a great deal of mixing exists within the street canyons. The buoyancy induced flow affects the air exchange between the street canyons and the free surface layer. Vertical air motions extend beyond the level of the street canyons causing instability to increase. It was found, that the stratification effects on the flow can be successfully predicted by the CFD numerical code.
\end{abstract}

Key words: Wind tunnel; street canyon; unstable stratification; pollutant dispersion; unstructured Cartesian grid

\section{INTRODUCTION}

Wind tunnels have been used for many years in the study of pollutant dispersion and the wind field, the large majority of which have been conducted under neutrally stratified conditions since stable or unstable boundary layer flows are both difficult and expensive to create. Flow and tracer two-dimensional experiments have been performed for neutral conditions around single or clusters of buildings. All wind tunnel experiments confirmed the presence of a large recirculation vortex within the urban canyon. The vortex strength and flow orientation depends on the building height (Garcia Sagrado et al., 2002, Chang, 2003) and affects the pollutant dispersion in the canyon (Gerdes et al., 1998). The effect of traffic on the wind flow and pollutant dispersion characteristics within a street canyon was also studied (Kastner-Klein et al., 2001) as well as the effect of the upwind conditions. A novel experiment for the study of the flow field and temperature within and above a single street canyon under stable and unstable conditions was conducted (Uehara et al., 2000) with the use of a Laser Doppler Anemometry along with a cold wire. Cavity eddies that arose in the street canyon tended to be weak when the atmosphere was stable and strong when unstable. When stability exceeded a certain threshold the wind speed in the street canyon dropped nearly to zero. On the other hand many numerical studies have been also reported in literature, determining wind flow and pollutant dispersion characteristics under neutral stratification in urban areas, which were validated against wind tunnel experimental data. The most used model is the standard $k-\varepsilon$ turbulence model (J. Ehrhard, and N. Moussiopoulos 2000, V. D. Assimakopoulos et al. 2003, X. Xie et al. 2006). Large-eddy simulations have also been conducted, providing reliable information on physical dispersion mechanisms and turbulence structures within urban canopy layers (A. Walton et al. 2002). Although LES seems to provide more accurate results than $k$ - $\varepsilon$, its computational cost makes its use prohibitive. The effects of ground-level heating; on the airflow structure has also been investigated (X. Xie 2006 et al. 2006, H. Huang et al. 2005). Within that frame the purpose of the present work is to study the characteristics of the flow field developed within and above urban street canyons, under neutral and unstable stratification conditions. The numerical code is validated with experimental results in a two dimensional experimental set up.

\section{EXPERIMENTAL DETAILS}

The experiment took place in the neutrally stratified open circuit wind tunnel of the Department of Environmental Physics and Meteorology, University of Athens. The test section of the wind tunnel is $1.2 \mathrm{~m}$ long, $0.4 \mathrm{~m}$ wide and $0.23 \mathrm{~m}$ high. For an empty working section the turbulence level of the free stream velocity, $400 \mathrm{~mm}$ from the inlet, is 0.5 percent. All the measurements were made with a free stream velocity $U o=3 \mathrm{~ms}^{-1}$ which corresponds to a Re number of 4000. A single building made of plexiglass sized $20 \mathrm{~mm} * 20 \mathrm{~mm}$ was placed at $700 \mathrm{~mm}$ from the test section entrance, which spanned the test section in order to create two-dimensional set up. Turbulence was created by a sand paper strip, $30 \mathrm{~mm}$ long which was placed in the entrance of the test section spanning the width of the tunnel. This created at a distance of $700 \mathrm{~mm}$ from the entrance a boundary layer $\delta=17 \mathrm{~mm}$ thick so that the ratio of boundary 
layer thickness $(\delta)$ to building height $(\mathrm{H})$ was equal to 8.5 . The forward face of the building was located $700 \mathrm{~mm}$ from the entrance of the test section. Mean velocity and turbulence measurements were taken using a triple hot wire constant-temperature anemometer (TSI) at several positions upwind, above and downwind the building. More specifically wind velocity and turbulence profiles were taken at $\mathrm{x}=15 \mathrm{H}$ and $5 \mathrm{H}$ upwind the forward face of the building, above the leading edge of the building and at $\mathrm{x}=2 \mathrm{H}$ and $5 \mathrm{H}$ downwind the leading edge of the building.

\section{NUMERICAL SIMULATIONS}

In the present study the averaged Navier-Stokes equations and $k-\varepsilon$ closure turbulence model system of equations is solved iteratively using the Simple algorithm modified for collocated grid arrangement. The grid employed is unstructured, locally refined, while the convection and diffusion terms are discretized using bounded second-order upwind (Papadakis, G. and Bergeles G., 1995). The latter was found to be very stable; while the algorithm converged without any under-relaxation of the source terms of the scheme. A flow solver for unstructured Cartesian grid embedded in space with oblique boundaries is developed. The set of linear equations is solved iteratively using a TDMA solver. The pollutants are simulated numerically, by introducing heat sources, avoiding the buoyancy effect. Those sources are defined applying Dirichlet conditions for the pollutants placed at the bottom, in the middle of the street canyons. Thus, the developed thermal field due to the sources corresponds to pollutant distribution, following Reynolds analogy. The iterative procedure for all fields was continued until the sum of the absolute cell residuals for mass, momentum and energy was satisfied to within $1 \%$ of the total mass, momentum and energy fluxes, respectively, at the inflow boundary. Numerical computations were firstly carried out to examine the performance of the CFD code, under neutral conditions. A mesh consisting of 40330 grid points was applied to a computational domain of $1.2 \mathrm{~m} \times 0.23 \mathrm{~m}$. A coarse structured uniform grid $(140 \mathrm{x} 40)$ was telescopically refined upwind, above and downwind an isolated building, representing an open country configuration. Computational cells of the coarse grid were divided uniformly into $\left(2^{k}\right)^{2}$ parts, where $k$ is the refinement level. The maximum refinement level $k$ is equal to 3. In order to investigate neutral and unstable turbulent flow including several street canyons (Fig. 1), the spatial domain was telescopically refined consisting of 53.715 computational cells; in which the minimum control cell is $3.125 \times 10^{-2} \mathrm{H} \times 3.357 \times 10^{-2} \mathrm{H}$. A total number of 352.128 cells would be required for a uniform Cartesian grid consisting of the above control volume. It is evident that the local refinement method results in reduction of the computational time needed for the simulation. Secondly the neutral and unstable turbulent flow including several street canyons was studied. A telescopically refined grid consisting of 53715 grid points was applied in order to maintain the accuracy at high level, within the street canyons (Fig 1). Buildings with height $\mathrm{H}=20 \mathrm{~mm}$ were placed within the computational domain at equal distances keeping the aspect ratio of building height to canyon width (W), constant and equal to $1, \mathrm{~W} / \mathrm{H}=1$. A total number of seven buildings, representing an urban environment, forming 6 street canyons perpendicular to the flow were considered. Unstable thermal stratification was created by setting operating temperature $\mathrm{T}_{\infty}=30^{\circ} \mathrm{C}$, floor temperature $\mathrm{T}_{\mathrm{f}}=90^{\circ} \mathrm{C}$ and inlet velocity $\mathrm{U}_{\text {ref }}=1.5 \mathrm{~ms}^{-1}$. From the above statements, the bulk Richardson number, defined as: $\mathrm{R}_{\mathrm{b}}=\left(\mathrm{gH}\left(\mathrm{T}_{\infty}-\mathrm{T}_{\mathrm{f}}\right) / \mathrm{U}_{\text {ref }}^{2} \mathrm{~T}_{\infty}\right)$ is -0.17 .

\section{RESULTS AND DISCUSSION}

\section{Single building}

Figure 2 shows a comparison between experimental and numerical results, of the u-component normalized velocity profiles, at different longitudinal positions. Comparison of computed and measured results is satisfactory. Upwind the building $(x=-5 H)$ the velocity increases with height up to $\mathrm{z}=\mathrm{H}$ and then stabilizes. The wind speed increases rapidly with height above the isolated building, at $\mathrm{x}=0$, up to $\mathrm{z}=2.5 \mathrm{H}$; being almost $10 \%$ larger than the reference velocity. Downwind the obstacle a strong recirculation zone is observed up to $\mathrm{x}=7 \mathrm{H}$. It is noticed that the computed flow field upwind and downwind the building, under neutral stratification, is in good agreement with the experimental measurements. The normalized Turbulent Kinetic Energy (T.K.E) profiles measured in the wind tunnel with the use of a triple sensor hot wire at different longitudinal points are shown in Fig. 3. The turbulence decreases with height at all measurement points. The maximum values are observed downwind the building $(\mathrm{x}=5 \mathrm{H})$ while upwind the building at distance $\mathrm{x}=-15 \mathrm{H}$ turbulence is damped remaining constant with height.

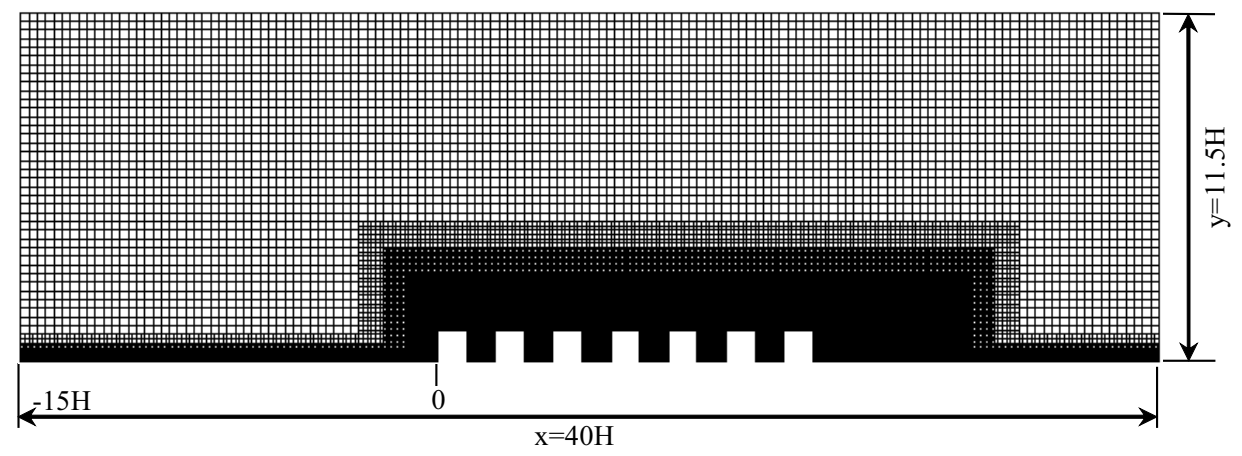

Figure 1. Computational grid - Telescopic refinement. 

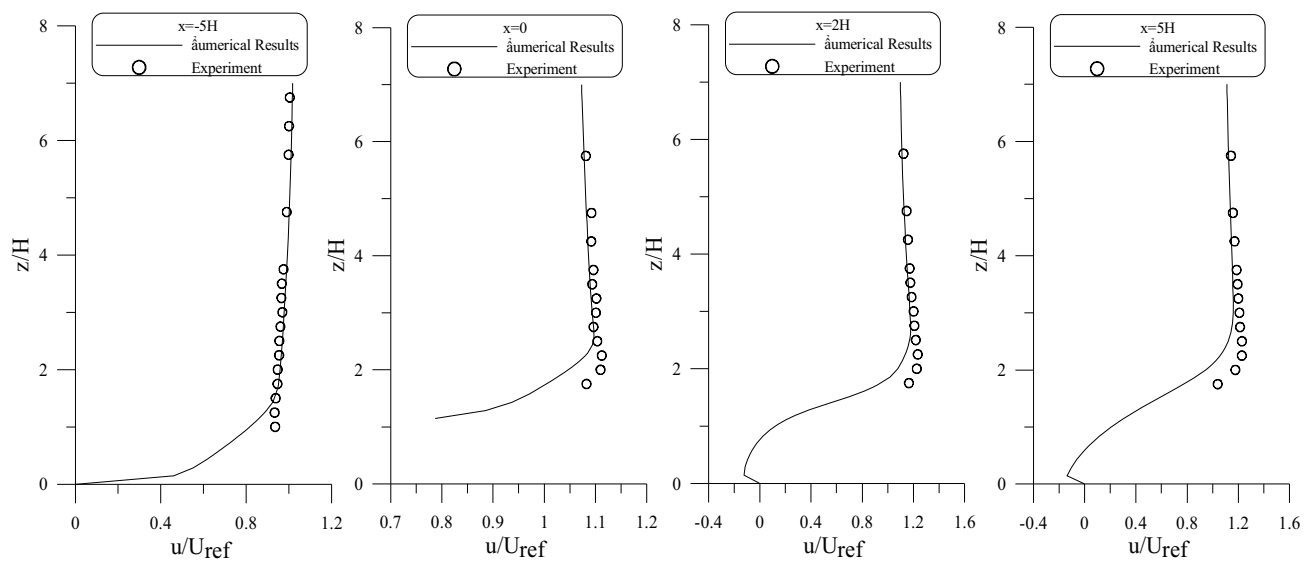

Figure 2. Comparison between experimental and numerical results under neutral stratification

\section{Urban Environment}

The numerical model is used to study the wind field under neutral and unstable conditions. Contours of the normalized vertical velocity components (W) within the street canyons for neutral and unstable stratification conditions are shown in Figure $4 \mathrm{a}$ and $4 \mathrm{~b}$. The effects of buoyancy on vertical air motions are observed under unstable stratification. The vertical component of the velocity is increased for the unstable case due to buoyancy effects within and above the canyon. Meanwhile, a noticeable ascent of the mean vertical velocities is observed, at the roof level of the street canyons. When the atmosphere is unstable, great deal of mixing in the street canyon exist, caused by higher diffusion due to buoyancy. The cavity eddy becomes stronger when the atmosphere is unstable. The pollutants' concentration has a direct correlation with the velocity field and stability of the atmosphere, inside the street. The vortex which is formed within the canyon pushes the pollutants in the left side of the street preventing the smoke from going out of the canyon. The pollutants that are allowed to go out from the street are carried by the flow towards the downstream building roof where important values of concentration are found.

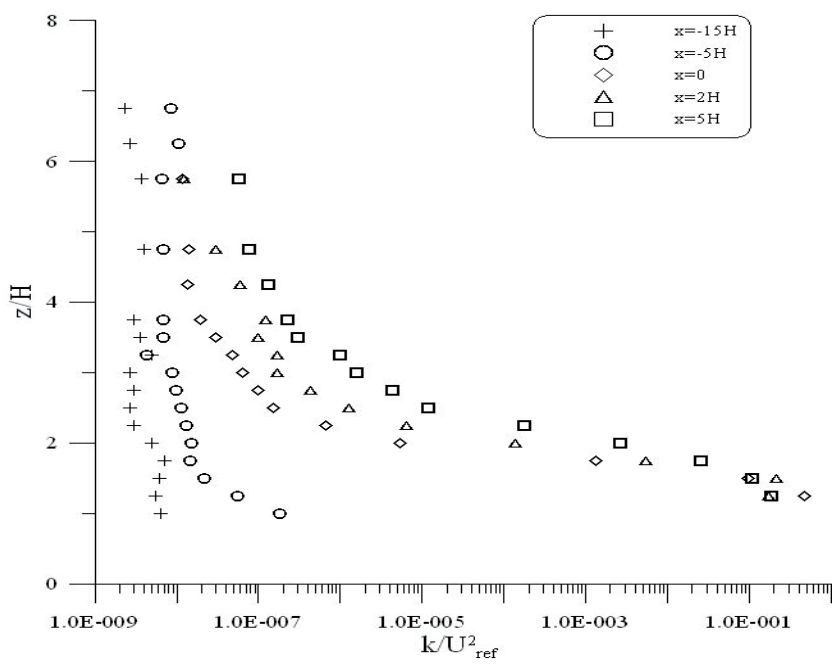

Figure 3. Normalized turbulent kinetic energy profiles.

\section{CONCLUSIONS}

The characteristics of the flow field developed around a single building and within and above urban street canyons, under neutral and unstable stratification conditions were studied both experimentally and numerically.

- The flow field computed around and above an isolated building under neutral stratification is in good agreement with the experimental measurements.

- Turbulence is enhanced above and downwind the isolated building under neutral conditions.

- Numerical simulations showed that the vertical component of the velocity is increased under unstable stratification conditions and the vortex established within the canyon is stronger for the multiple canyons case. 
- Pollutants concentration has a direct correlation with the velocity field and stability of the atmosphere, inside the street. The vortex which is formed within the canyon pushes the pollutants in the left side of the street preventing the pollutant from going out of the canyon. The pollutants that are allowed to go out from the street are carried by the flow towards the downstream building roof where important values of concentration are found.

Acknowledgements: This research project (PENED) is co-financed by E.U.-European Social Fund (75\%) and the Greek Ministry of Development-GSRT (25\%).

\section{REFERENCES}

Assimakopoulos, V.D., ApSimon, H.M. and Moussiopoulos, N. 2003: A numerical study of atmospheric pollutant dispersion in different two-dimensional street canyon configurations. Atmospheric Environment, 37, 40374049.

Chang, C. and Meroney R. N. 2003: Concentration and flow distributions in urban street canyons: wind tunnel and computational data. Journal of Wind Engineering and Industrial Aerodynamics, 91 (9), 1141-1154.

Gerdes, F. and Olivari, D. 1998: Analysis of pollutant dispersion in an urban street canyon. Journal of Wind Engineering and Industrial Aerodynamics, 82 (1-3), 105-124.

Huang, H., Ooka, R. and Kato, S.: 2005: Urban thermal environment measurements and numerical simulation for an actual complex urban area covering a large district heating and cooling system in summer. Atmospheric Environment, 39, 6362-6375.

Kastner-Klein, P., Plate, E. and Fedorovich, E. 2001: A wind tunnel study of organized and turbulent air motions in urban street canyons. Journal of Wind Engineering and Industrial Aerodynamics, 89 (9), 849-861.

Papadakis, G. and Bergeles G. 1995: A Locally Modified Second Order Upwind Scheme for Convection Terms Discretization. International Journal of Numerical Methods for Heat \& Fluid, 5, 49-62.

Sagrado, A. P. G., Beeck, J., Rambaud, P. and Olivari, D. 2002: Numerical and experimental modeling of pollutant dispersion in a street canyon. Journal of Wind Engineering and Industrial Aerodynamics, 90 (4-5), 321-339.

Uehara, K., Murakami, S., Oikawa, S. and Wakamatsu S. 2000: Wind tunnel experiments on how thermal stratification affects flow in and above urban street canyons. Atmospheric Environment, 34 (10), 15531562.

Walton, A. and Cheng, A.Y.S. 2002: Large-eddy simulation of pollution dispersion in an urban street canyon-Part II: idealized canyon simulation. Atmospheric Environment, 36, 3615-3627.

Walton, A., Cheng, A.Y.S. and Yeung, W.C. 2002: Large-eddy simulation of pollution dispersion in an urban street canyon-Part I: comparison with field data. Atmospheric Environment, 36, 3601-3613.

Xie, X., Liu, C., Leung D.Y.C. and Leung M.K.H.: 2006: Characteristics of air exchange in a street canyon with ground heating. Atmospheric Environment, 40, 6396-6409. 


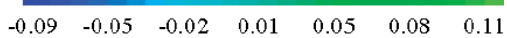

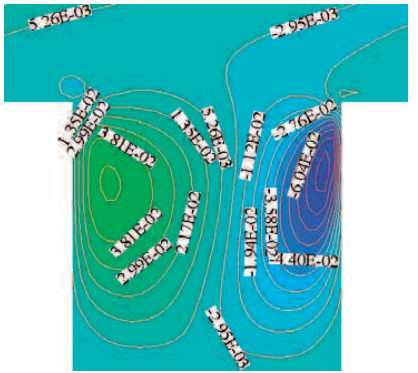

a) First canyon

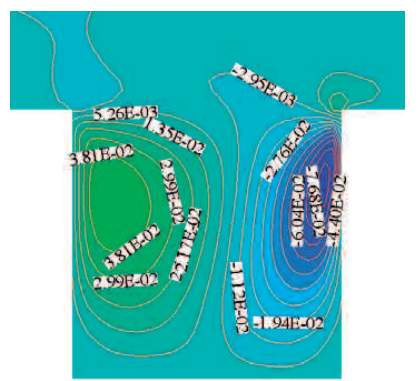

b) Third canyon

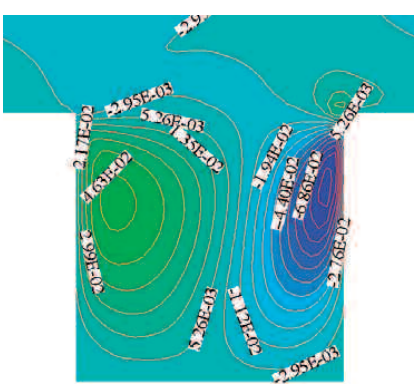

c) Last canyon

Figure 4a. Contour of $\mathrm{W} / \mathrm{U}_{\text {ref }}$ within the street canyons, under neutral stratification

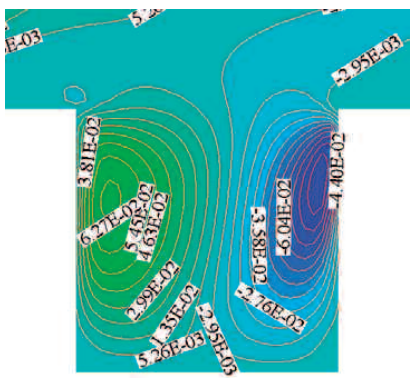

a) First canyon

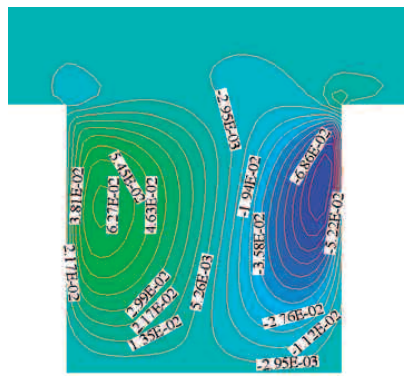

b) Third canyon

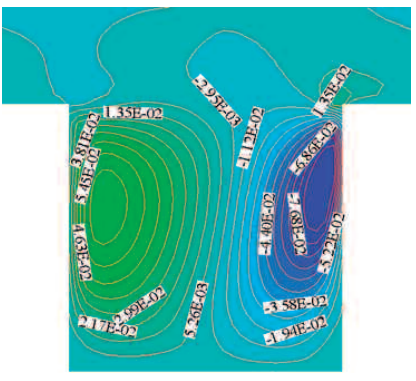

c) Last canyon

Figure $4 \mathrm{~b}$. Contour of $\mathrm{W} / \mathrm{U}_{\text {ref }}$ within the street canyons, under unstable stratification

\section{$0.010 \quad 0.0190 .0370 .0720 .1390 .2680 .5181 .000$}

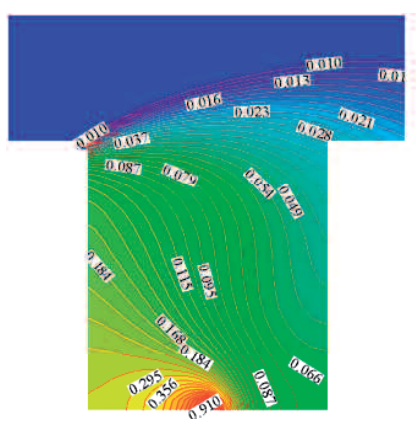

a) First canyon

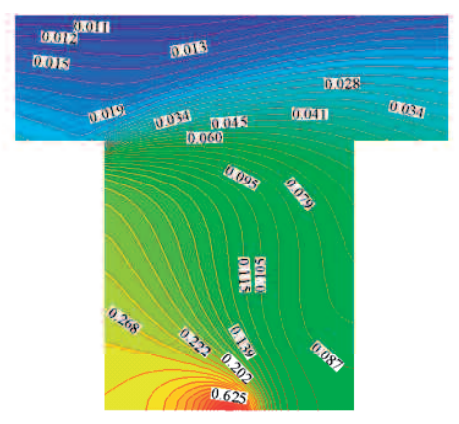

b) Third canyon

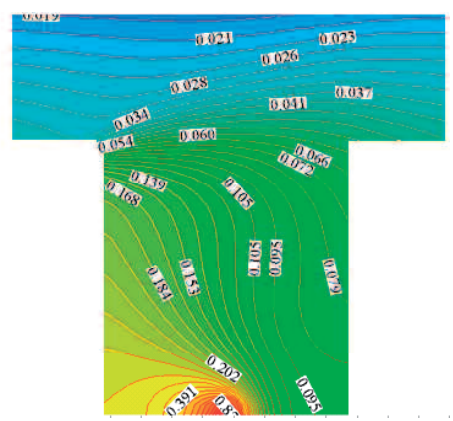

c) Last canyon

Figure 4c. Contour of normalized pollutant distribution, under neutral stratification

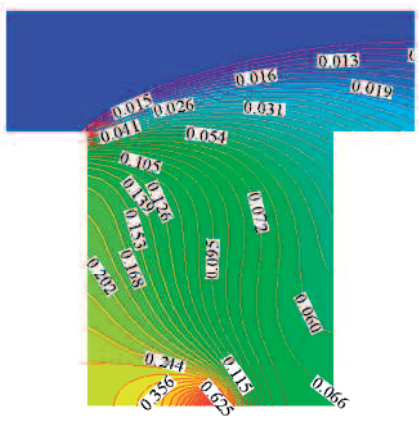

a) First canyon

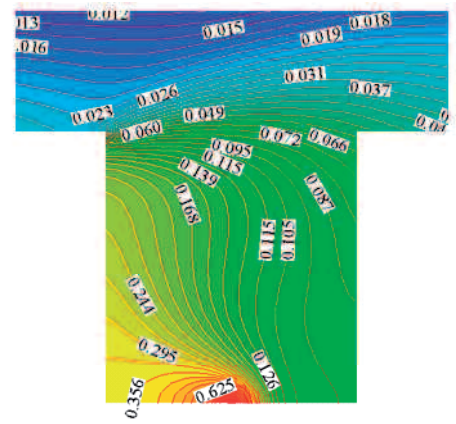

b) Third canyon

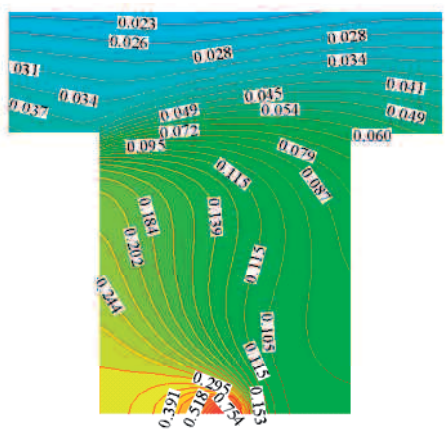

c) Last canyon

Figure 4d. Contour of normalized pollutant distribution, under unstable stratification 